\title{
Supporting First Person Shooter Games with Competing Traffic in 802.11e MAC
}

\author{
Hanghang Qi, David Malone and Ashwin Murali \\ Hamilton Institute \\ National University of Ireland, Maynooth, Ireland \\ Email:\{hanghang.qi,david.malone\}@nuim.ie
}

\author{
Dmitri Botvich \\ TSSG \\ Waterford Institute of Technology, Ireland \\ Email:dbotvich@tssg.org
}

\begin{abstract}
As real-time multi-player game is one of the most popular network applications nowadays and 802.11 wireless networks are fast developed and widely used, it is common that games are played over 802.11 networks. However, games performance can be degraded competing with background traffics. In this paper, we propose a Application Divided Resource Allocation (ADRA) scheme based on 802.11e to support games in mixed traffic 802.11 networks. Parameter selection and performance improvement are analyzed with our models. Testbed verification validates the effectiveness of our scheme and analysis which provides great improvement to games and also sustains the performance of the background traffics.
\end{abstract}

\section{INTRODUCTION}

Real-time multi-player game such as FPS game is one of the most popular network applications and it requires network requirement such as low delay, jitter etc [1], [2]. IEEE 802.11 wireless local area networks are widely used so real-time multi-player games are more and more played over 802.11 wireless networks.

Heavy background traffic can degrade game performance mainly because of large queuing delay, jitter and loss etc. In 2005, the 802.11e extension was approved to support QoS. The 802.11e MAC enables the values of four MAC parameters $D I F S$ (called $A I F S$ ), $C W_{\min }, C W_{\max }$ and $T X O P$ to be set on a per class bases for each station, and currently they require four classes be supported. In this paper, we proposed an Application Divided Resource Allocation (ADRA) scheme based on the 11e virtual queues and MAC parameters to support game with other traffic.

Related works include [3], [4] and [5]. [3] propose a scheme at the IP-layer rather than a MAC-layer to support games in WLAN. [5] measures the capability of 802.11g for multiplayer online games.

\section{Application Divided Resource Allocation SCHEME(ADRA)}

The ADRA scheme is described as follows dealing with internal and external congestion respectively:

1) Separate game traffic and the other traffic into 2 different queues based on the virtual queues with $11 \mathrm{e}$ which greatly reduces the buffering delay of game traffic when sharing the network with bulk TCP traffic for station and AP particularly.
2) Give game traffic and other traffic different MAC parameters based on the performance requirement of games and other traffic.

\section{Modeling and Parameters Selection}

By using the Bianchi model or Renewal Reward approach, we can calculate the performance of delay and throughout for games and competing traffic. We have the 11e Parameters as the model parameters. Fig. 1 gives an example of the delay result for different parameters: $c w_{1}, m_{1}$ and $A I F S_{2}$, where $c w_{1}=c w_{\min 1}$ and $c w_{\max }=2^{m} c w_{\min }$ with other parameters fixed in table I. It shows that, as $c w_{1}$ reduces for game, it largely reduces the delay for game and mildly increases the delay for background traffic. $m_{1}=3$ gives better performance than $m_{1}=5$. $A I F S_{2}=6$ gives better performance than $A I F S_{2}$ is smaller or larger.

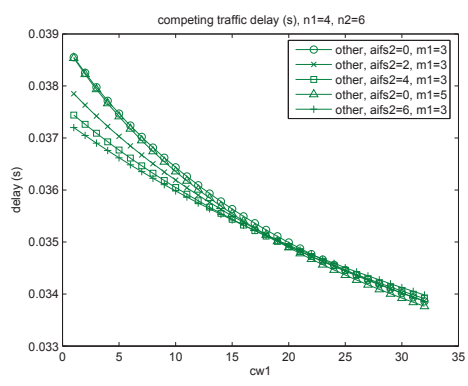

(a) competing traffic

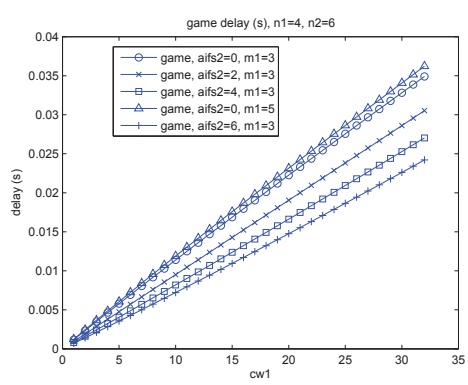

(b) game traffic

Fig. 1. RTT delay of competing traffic and game traffic

For other values of $n_{1}, n_{2}$, the same method can be used to determine 11e parameters. For number of game flows from 
2 to 6 and number of competing flow from 2 to 8, parameters shown in table I give the best game performance and when the game flows is smaller than $14, c w_{1}=1$ continues to give the best performance.

\section{TESTBED VERIFICATION}

Our testbed consists 4 Dell PCs and 7 Soekris boxes behaving as stations for generating background traffic. The access point and the stations use Atheros-based wireless cards and the MadWiFi driver. The Quake IV demo on Linux is used. MGEN and Iperf are used to generate UDP and TCP traffic from both stations and the APs as upload and download traffic respectively. Tcpdump is used to record traffic. The ping command is used to get the Round Trip Time (RTT), as the measurement of the delay.

In our tests we compare two configurations. First, the basic 802.11 MAC is used without any parameter adjustment. We implement this by putting all packets in the 'BE' queue. Second, we implement the ADRA scheme. We use two 802.11e queues at each station (Table I). We allocate packets to queues based on the packet size: packet smaller than 200 bytes are put into the game queue while other packets use BE queue. ICMP (ping) packet is also in the same queue as games as its size is also smaller than 200 bytes.

TABLE I

EDCA PARAMETERS SETTING

\begin{tabular}{|l|l|l|l|l|}
\hline AC & $C W_{\min }$ & $C W_{\max }$ & AIFSN & TXOP(ms) \\
\hline competing traffic & 32 & 1024 & 6 & 0 \\
game traffic & 1 & 8 & 0 & 2.2 \\
\hline
\end{tabular}

Fig. 2 shows results for networks with 6 game stations and between 2-8 other stations (including the AP) sending saturated UDP traffic with downloading and uploading mixed traffic. We see that without ADRA the delays measured are tens of milliseconds for both game and other traffic. With ADRA scheme we see that the average delay for the competing traffic increases by around $20 \%$, however the delay for the game traffic is dramatically reduced, by around $90 \%$.

Fig. 3 shows similar results for TCP traffic. Without ADRA we see RTT is above $90 \mathrm{~ms}$ and in practice the game almost unplayable. With ADRA the RTT drops to around $4 \mathrm{~ms}$. This benefit mainly comes from the virtual queue allocation, because the game packets no longer share the full queue with the TCP packets.

\section{CONCLUSiOns AND FUture WORKS}

In this paper, we proposed a scheme called application driven resource allocation (ADRA) at the 802.11e MAC layer to support First Person Shooter (FPS) games in the presence of competing traffic. The scheme addresses both internal contention at nodes and external contention on the 802.11 medium by using virtual queues and different MAC parameters. We can achieve larger throughputs and small delays for FPS games with background competing traffic. We present testbed measurements and confirm the effectiveness of ADRA. Future

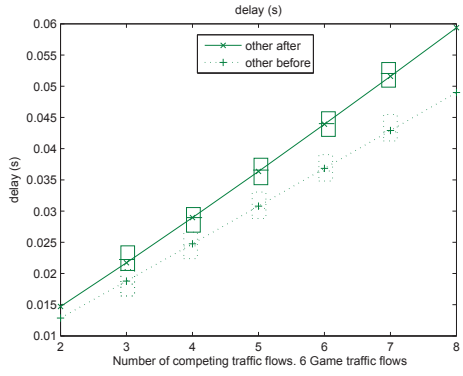

(a) delay for competing traffic

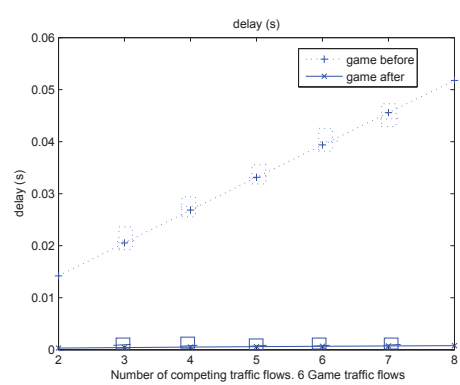

(b) delay for game traffic

Fig. 2. Testbed results for 6 game flows vs. 2-8 saturated flows

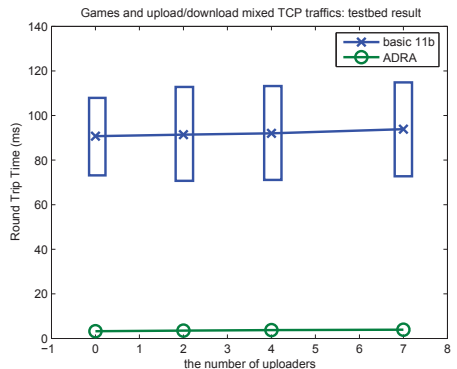

Fig. 3. Testbed TCP traffic result

works include how to identify game traffic and automatically parameters turning.

\section{REFERENCES}

[1] M. Claypool and K. Claypool, "Latency and player actions in online games,” Commun. ACM, vol. 49, 2006.

[2] A. F. Wattimena, R. E. Kooij, J. M. van Vugt, and O. K. Ahmed, "Predicting the perceived quality of a first person shooter: the Quake IV G-model,” in NetGames '06, NY, USA, ACM.

[3] B. Carrig, D. Denieffe, and J. Murphy, "Supporting first person shooter games in wireless local area networks," in Personal, Indoor and Mobile Radio Communications, 2007. IEEE 18th International Symposium on, Sept.

[4] T. Nguyen and G. Armitage, "Quantitative assessment of ip service quality in 802.11b and docsis networks," in Proceedings of the Australian Network and Telecommunications Conference 2004.

[5] Y. E. Liu, J. Wang, M. Kwak, J. Diamon, and M. Toulouse, "Capability of IEEE 802.11g networks in supporting multi-player online games,” in Consumer Communications and Networking Conference, 2006. 3rd IEEE, vol. 2, pp. 1193-1198. 